\title{
Proton Conductive and Low Methanol Permeable PVA- based Zwitterionic Membranes
}

\author{
Liang Gao*, Tengfei Kong, Guoqiang Guo, Yanping Huo*
}

Keywords: polyzwitterion, proton exchange membrane, acid-base interaction, methanol barrier.

\section{Abstract:}

A desirable membrane for direct methanol fuel cell (DMFCs) must both conduct protons and function as a methanol barrier. However, it remains challenge to balance the proton conduction and methanol permeability. As a commonly used pervaporation membrane, PVA has high selectivity for water over methanol, but its proton conductivity is too low for DMFCs applications. To improve conductivity, efforts have been predominantly focused on direct incorporation of strongly acidic groups, such as sulfonated or phosphoric groups on the PVA chain. This strategy can result in improvement in the proton conduction. However, the performance of acid modified PVA is compromised due to the strong charge-charge repulsion of attached groups, which results in the increased fraction free volume that facilitates methanol permeation. We attempt to minimize the tradeoff between proton conduction and methanol permeability by modifying PVA with zwitterionic groups. This is achieved by functionalizing PVA with pendent zwitterionic groups (PVA-ZW-n) at controllable substitution degree $(5<\mathrm{n}<20)$. The investigation of their $1 \mathrm{H}$ NMR spectra and glass transition temperatures $\left(\mathrm{T}_{\mathrm{g}}\right)$ suggests that there is strong electrostatic interaction in the PVA-ZW-20 polymer, as confirmed by the observed proton spatial distribution in $\mathrm{NMR}_{2}$ and increased $\mathrm{T}_{\mathrm{g}}$ along with the increase of substitution degree in DSC. The synthesized PVA-ZW-20 can be 
easily casted into mechanically stable membranes with modest proton conductivity. After incorporation with (3-Mercaptopropyl)trimethoxysilane (MPTMS) and successive oxidation, the synthesized PVA-ZW-20-Si exhibits lower methanol permeability and reasonably higher proton conductivity compared to Nafion 117. A series of control experiments revealed that the superior performance of PVA-ZW-20-Si could be related to the presence of zwitterionic groups, which result in enhanced packing density of polymer chain and more efficient proton dissociation.

\section{Introduction}

Direct methanol fuel cells (DMFCs) are widely regarded as an ideal power supply for portable devices due to their high energy power density and quick start-up. ${ }^{1}$ Methanol has several distinct advantages over other types of fuels, e.g. ease of handling and high volumetric energy density $\left(4.75 \mathrm{kWh} \mathrm{L}^{-1}\right){ }^{2-3}$ However, methanol tends to cross the membrane and reacts with $\mathrm{O}_{2}$ at cathode, releasing energy as heat. This methanol crossover results in the establishment of mixing potential at the cathode, leading to degradation in overall performance. ${ }^{4}$ Therefore, eliminating the methanol crossover is of great importance for DMFCs, ${ }^{4-7}$ and mainly relies on the development of advanced proton conduction membrane (PEM), which acts as a medium for fast proton transportation while at the same time functions as a barrier for eliminating crossover of methanol. Currently, state of the-art candidates mainly include Nafion series and different sulfonated polyetherketones. ${ }^{7-9}$ Although these membranes possess high proton conductivity and good mechanical stability, serious methanol crossover still limits them from practical applications on DMFCs.

Current research reveals that reduced water swelling of membrane is desirable for methanol blockage. ${ }^{10-11}$ Therefore, intensive efforts have been made to control the hydration degree of PEMs by embedding hydrophobic species. However, insufficient hydration adversely influences proton conduction due to the lack of proton transportation medium. Hence, the trade-off between methanol permeability and proton conductivity is a common concern when employing the hydration-control strategy. ${ }^{12}$ 
Alternatively, a type of ionic "barrier" constituted by acid-base interaction has been recognized to be effective in optimizing the balance between methanol permeability and proton conductivity. ${ }^{13-17}$ The ionic "barrier" can be formed when the charged acidic and basic groups react to form a loosely connected network. This network contributes to the morphological stability and block methanol permeation; while at the same time, the positively charged basic groups in this network can electrostatically promote the proton dissociation and facilitate the proton transportation. ${ }^{13-15}$ In most previously reported cases, the acid-base interaction is established by incorporating isolated acidic and basic compounds. Among these cases, the simplest is to dope small molecules bearing either basic or acidic sites, e.g. $\mathrm{H}_{3} \mathrm{PO}_{4}$ /polybenzenimidazole (PBI) membrane. However, small molecule $\mathrm{H}_{3} \mathrm{PO}_{4}$ is likely to be leached out or dissolved into water/methanol, ruling out the possibility for DMFCs application. Blending of acidic/basic polymer would be a more practical choice than polymer/small molecule composites. ${ }^{14-17}$ However, blending strategy seriously limits the scope of suitable candidates, since quiet a lot of polymers are immiscible due to the unfavorable mixing entropy. ${ }^{18}$ In some cases, intensive energy input (e.g. heating) or post-treatment (e.g. crosslinking) ${ }^{14-16}$ is necessary to invoke the mixing. The general validity of ionic barrier strategy relies on the development of suitable materials possessing opposite charged groups.

In order to circumvent these abovementioned drawbacks by employing isolated acidic and basic compounds, the motivation of this work is to explore the potential of utilizing zwitterionic materials to achieve the establishment of acid-base interaction. Zwitterionic materials contain base-type (e.g. $\left.-\mathrm{NR}_{3}{ }^{+}\right)$and acid-type (e.g. $\left.-\mathrm{SO}_{3}{ }^{-},-\mathrm{COO}^{-}\right)$ charged groups in one single neutral molecule. ${ }^{19}$ Being characteristic of high dipole moment and high charge density, polyzwitterions have a wide range of applications including ion exchange, ${ }^{20}$ chelation for scavenging trace metals from drinking water $^{21}$,antifouling/antibacterial function ${ }^{22-23}$ and stimulation responsive adhesion. ${ }^{24}$ Doug MacFarlane et al., ${ }^{25}$ first demonstrated that polyzwitterion can work as Li-ion dissociator to enhance $\mathrm{Li}$ ion conductivity. The similar effects of zwitterions on promoting the proton conductivity were reported. ${ }^{26-28}$ 
Except for enhancement in proton conductivity, similar to the principle proposed for the aforementioned acid-base interaction, the high-density of opposite charged groups in polyzwtterionic membrane can promote the strong chain-chain interaction and dramatically compress the fraction volume, thus swelling is effectively controlled as compared to these counterparts containing only sulfonated groups. ${ }^{29-31}$ Therefore, polyzwitterion seems to be capable of establishing the desired acid-base network via electrostatic interaction to reduce the tradeoff between methanol blockage and enhancement in proton transport. Distinctly different from blending mechanism, whereby th acidic and basic sites are physically mixed together; zwitterionic polymer naturally embeds acid and base in one macromolecule, thus eliminating the concerns of immiscibility.

Here, a series of novel PVA-based polyzwitterions were synthesized by partially modifying the PVA hydroxyl groups: first, N1,N1-diethylethane-1,2-diamine (DEEDA) was coupled to give PVA-DEEDA-n by carbonyldiimidazole (CDI)-mediated couplings via carbamate bonds. ${ }^{32}$ The choice of polyvinyl alcohol (PVA) as parent polymer is due to its well-known alcohol resistance. ${ }^{33}$ PVA-DEEDA-n was then further reacted with 1,3propane sultone to add charged zwitterionic groups $\left(-\mathrm{NEt}_{2}-\left(\mathrm{CH}_{2}\right)_{3}-\mathrm{SO}_{3}\right)$ to form PVA$\mathrm{ZW}$. The added amount of zwitterionic group was tuned by changing the feed ratio of CDI. The structure of the resulting polymers was characterized by using $1 \mathrm{H}$ NMR and FTIR. PVA-ZW both with and without (3-Mercaptopropyl)trimethoxysilane (MPTMS) incorporation were casted into membrane. The thermal properties, $\mathrm{MeOH} / \mathrm{H}_{2} \mathrm{O}$ selective uptake, swelling behavior, methanol permeability and proton conductivity of the obtained membranes were systematically investigated and compared with benchmark membrane Nafion 117. Furthermore, the zwitterion effects were highlighted by comparing the performance of MPTMS doped pristine PVA and zwitterion modified PVA-ZW membrane. The current results suggest the PVA-ZW-20-Si membrane shows lower methanol crossover and higher proton conduction than its counterpart PVA-Si, indicating the key role of zwitterionic group. 
Figure 1. (1) Schematic representation for the preparation of zwitterion functionalized PVA (PVA-ZW).

Step_1: the hydroxyl groups on PVA is activated by carbonyldiimidazole (CDI); Step 2: CDI activated PVA reacts with $\mathrm{N}^{1}, \mathrm{~N}^{1}$-diethylethane-1,2-diamine (DEEDA) to obtain DEEDA modified PVA; Step 3: Ringopen reaction happens between propane sultone and amine group on DEEDA modified PVA to give zwitterionic group functionalized PVA (PVA-ZW); (2) Schematic diagram of the proposed mechanism of the prepared membrane for proton conduction and methanol blockage.

\section{Experimental}

2.1 Materials. Polyvinyl alcohol (PVA, Aldrich, average MW=16,600, hydrolysis degree=99\%,), N1,N1-diethylethane-1,2-diamine (DEEDA, Acros, purity>98\%), carbonyldiimidazole (CDI, Dikemann (China), purity>99\%), 1,3-propane sultone, mercaptopropyl methyldimethoxysilane (MPDMS) were used as received. All aqueous solutions were prepared from mill-Q 18M DI. water. Dimethyl sulfonate (DMSO) and Dimethylformamide (DMF) solvents were vacuum-distilled to remove water.

2.2 Synthesis of PVA bearing zwitterionic group. An efficient method for appending zwitterions group to PVA under mild conditions via three-step reactions is present in the reaction route of Fig. 1 and described below:

Step 1. The relatively inert -OH group of PVA can be activated based on the strategy reported previously. ${ }^{32}$ The substitution degree of $-\mathrm{OH}$ group is positively proportional to the feeding amount of activation agents, CDI (See Fig. 2). Typically, for a $20 \%$ substitution degree, 5g PVA was dissolved by $200 \mathrm{~mL}$ dry DMSO in a $500 \mathrm{~mL}$ round bottom flask by heating. The PVA solution was cooled down to room temperature and 9.2g CDI was added. The obtained mixture of PVA and CDI was stirred for $3 \mathrm{~h}$ at room temperature.

Step 2. Step 1 allows PVA to be activated by CDI. Afterwards, stoichiometric N1,N1diethylethane-1,2-diamine (DEEDA) ( $8 \mathrm{~mL}$ for $20 \%$ substitution degree) was added to CDI activated PVA drop-by-drop. Stirring was maintained for additional 20h. Then, 50 
$\mathrm{mL}$ of $28 \%$ ammonia solution was added and the solution mixture was stirred for $1 \mathrm{~h}$ to remove non-replaced CDI. The product, namely PVA-DEEDA, was precipitated out by adding 10-fold acetone and purified by dialysis using DI water for 3 days.

Step 3. The purified white PVA-DEEDA was dissolved in DMF and reacted with stoichiometric propane sultone at $22^{\circ} \mathrm{C}$ for 3 days. No heating was applied to avoid esterification reaction between sulfonate and hydroxyl groups of PVA. The final product PVA-ZW-n (n stands for substation degree and is the mole ratio of [pendant zwitterionic group] to the $[\mathrm{OH}]$ of parent PVA) was precipitated out and purified by dialysis by following the similar operation in step 2 .

2.3. PVA-ZW Polymers Analysis and Measurement. 1H nuclear magnetic resonance (NMR) spectra of various PVA derivatives PVA-DEEDA and PVA-ZW were recorded using Bruker 400M equipment. Deuterated water was used as solvent, and the $\mathrm{H}_{2} \mathrm{O}$ signal at $4.79 \mathrm{ppm}$ was taken as chemical shift reference. FTIR spectra (KBr pellet) was recorded on a Shimadzu $8400 \mathrm{~S}$ at a resolution of $4 \mathrm{~cm}^{-1}$. CHNS element analysis was performed on an Elemental Analyzer Perkin Elmer Series II 2400 to determine the content of sulfonate group. To study the proton dissociation of PVA-ZW polymers, the $\mathrm{pH}$ of aqueous solution of PVA-ZW at $10 \mathrm{wt} \%$ concentration is measured by using Shanghai Leici PHS-3b pH meter.

2.4 Preparation of Membrane Films. Membranes were prepared via solution casting method. The casting procedure is shown in Fig. S1 in ESI: To prepare PVA, PVA-ZW, PVA-ZW-Si and PVA-Si membranes, 0.2 g neat PVA, PVA-ZW-X (X=5 or 20), MPDMS/PVA(1:1 wt/wt\%) and MPDMS/PVA-ZW-20 (1:1 wt/wt\%) were dissolved in $5 \mathrm{~mL}$ water to obtain viscous casting solutions, respectively (see Table 1). The casting solution was then poured into a round Teflon mould with diameter of ca. $4 \mathrm{~cm}$. and dried inside a fume hood at room temperature. Subsequently, the membranes were annealed at $80^{\circ} \mathrm{C}$ for $1 \mathrm{~h}$. To further enhance the mechanical stability, the annealed membranes were immersed into a $0.01 \mathrm{M} \mathrm{HCl}$ acetone solution containing $2 \mathrm{wt} . \%$ glutaraldehyde for $2 \mathrm{~h}$ at $60^{\circ} \mathrm{C}$ to crosslink the aldehyde with residual $-\mathrm{OH}$ groups of PVA. Following the crosslinking process, the MPDMS/PVA-ZW or MPDMS/PVA membranes were immersed in the aqueous solution of $30 \%$ hydrogen peroxide at room temperature for $48 \mathrm{~h}$ 
and then soaked in $1 \mathrm{M} \mathrm{H}_{2} \mathrm{SO}_{4}$ to fully convert the mecapto (-SH) group into the sulfonic acid. $^{34}$

\subsection{Water Uptake, Water/methanol Selectivity, Swelling Ratio, Methanol Crossover and Mechanical Testing.}

To determine the water uptake membrane was dried over P2O5 for at least 3days, weighed and immersed into beakers containing DI water. After $72 \mathrm{~h}$, the membrane was taken out, quickly wiped by using tissue to remove the water on the surface, and weighed. Respective water uptakes of the prepared membranes were calculated from the following Eq. (1):

$$
\text { Water uptake }(\%)=\left(\mathrm{W}_{\text {wet }}-\mathrm{W}_{\mathrm{dry}}\right) \times 100 / \mathrm{W}_{\mathrm{dry}}
$$

where $\mathrm{W}_{\text {wet }}$ represents the respective weights of wet membranes soaked in DI water for $24 \mathrm{~h}$, and $\mathrm{W}_{\mathrm{dry}}$ is the respective weights of dry membranes.

Methanol adsorption from the methanol-water mixture was measured at room temperature (ca. $23^{\circ} \mathrm{C}$ ). The membranes were thoroughly dried in a desiccator over $\mathrm{P}_{2} \mathrm{O}_{5}$ for $72 \mathrm{~h}$. Afterwards, these membranes were soaked in excess methanol-water solution and kept equilibrated for another $72 \mathrm{~h}$ (Fig. S2). The methanol concentration of pristine methanol-water solution was calculated according to Eq. S1 as described in ESI. Upon contacting the membranes, the concentration change of $\mathrm{MeOH}$ was analyzed using GCFID, which has been carefully calibrated before using with $n$-propanol alcohol as internal standard (see Fig. S3). During the test, the column oven was set to be $120^{\circ} \mathrm{C}$, and detector temperature was $300^{\circ} \mathrm{C}$.

Methanol permeability was measured by a diffusion cell, shown in Fig. S4. The cell consists of two chambers separated by a membrane with effective area $\mathrm{A}_{\mathrm{d}}$. Initially, one chamber (A) was filled with $100 \mathrm{~mL} 30$ wt. $\%$ methanol/water (methanol/(water+methanol), ca. 9.2 M) mixture and the other chamber (B). Methanol penetrates through the membrane driven by the concentration gradient, thus methanol concentration in chamber B increases with time. The time duration is $72 \mathrm{~h}$.

The methanol permeability $(\mathrm{P})$ was estimated by the equation: 


$$
P=\frac{1}{A_{d}} \frac{C_{B}(t)}{C_{A} \times\left(t-t_{0}\right)} V_{B} l
$$

where $A_{d}$ is the effective permeation area, $C_{A}$ is the initial methanol concentration of chamber $A ; C_{B}(t)$ is the methanol concentration of chamber $B$ at time $t ; t_{0}=0$ supposing no time lag; 1 is the wet membrane thickness by using vernier caliper.

The swelling ratio in $0.52 \mathrm{~g} / \mathrm{mL} \mathrm{MeOH} /$ water mixture is used to evaluate the dimensional stability of the membranes during DMFCs application. The swelling ratio of a round membrane can be calculated as follows:

$$
S_{\mathrm{v}}=\frac{R_{w e t}-R_{d r y}}{R_{d r y}} \times 100 \%
$$

where $\mathrm{R}$ is the radius of membrane measured by vernier caliper in either dry or wet sates as indicated by the subscript.

The tensile strength and elongation at break experiments were conducted with ca.75\% humidity on a Labthink XLW (PC) at the strain rate of $25 \mathrm{~mm} / \mathrm{min}$ and a $50 \mathrm{~N}$ load cell at ca. $25^{\circ} \mathrm{C}$.

\subsection{Ion Exchange Capacity (IEC), Proton Conductivity and Oxidative Stability.}

The IEC of each membrane was studied by using conventional titration methods. Before testing, the membranes were sealed in a desiccator and dried over $\mathrm{P}_{2} \mathrm{O}_{5}$. The dried membrane is then equilibrated in excess $1 \mathrm{M} \mathrm{NaCl}$ to fully exchange protons out from the membrane to the solution. Subsequently, the solution is titrated with $10 \mathrm{mM} \mathrm{NaOH}$ using $0.1 \%$ phenolphthalein solution in ethanol/water $(90 / 10 \mathrm{wt} / \mathrm{wt})$ as indicator. The membrane retained in the solution during the whole titration process.

$$
I E C=\frac{0.01 m M \times V_{N a O H}}{W_{d r y}}
$$

Conductivity of membranes was measured by two-probe through plane and in plane AC impedance spectroscopy using a frequency ranging from $1 \mathrm{M}$ to $1 \mathrm{~Hz}$, and a perturbation of $10 \mathrm{mV}$, on a Solatron 1260 gain phase analyzer. The home-made test cell is shown in 
Fig. S5. Before measurement, all membranes have been equilibrated in $0.1 \mathrm{M} \mathrm{H}_{2} \mathrm{SO}_{4}$ and then DI water for $24 \mathrm{~h}$. The membrane was then washed with water to remove excess $\mathrm{H}_{2} \mathrm{SO}_{4}$. During the proton conduction tests, the whole test cell was immersed into DI water to maintain full hydration. Membrane conductivity $(\sigma)$ was calculated by the following equation according to previous literature:

$$
\sigma=\frac{l}{R A}
$$

Where 1 is the thickness of wet membrane for through-plane measurement or the gap between electrodes for in-plane measurements, A is the membrane cross-sectional area in case of the in-plane setup and the area of the electrodes in case of the through-plane setup. and $\mathrm{R}$ is the resistance value at high frequency or the intercept with the real part of impedance.

The activation energy $\left(\mathrm{E}_{\mathrm{a}}, \mathrm{kJ} \mathrm{mol}^{-1}\right)$, which is associated with energy barrier of proton movement, was obtained for each membrane based on the Arrhenius equation:

$$
\ln \sigma=\frac{-E_{a}}{R T}
$$

Here, $\sigma$ is the proton conductivity (in $\left.\mathrm{S} \mathrm{cm}^{-1}\right), \mathrm{R}$ is the universal gas constant $(8.314 \mathrm{~J}$ $\left.\mathrm{mol}^{-1} \mathrm{~K}\right)$, and $\mathrm{T}$ is the absolute temperature $(\mathrm{K})$.

The oxidative stability was evaluated by soaking the membranes in Fenton's reagent $\left(3 \% \mathrm{H}_{2} \mathrm{O}_{2}\right.$ containing $\left.2 \mathrm{ppm} \mathrm{FeSO}_{4}\right)$ at $80^{\circ} \mathrm{C}$. To this end, the membrane was thoroughly dried over $\mathrm{P}_{2} \mathrm{O}_{5}$ at $30^{\circ} \mathrm{C}$ before the weight was recorded ( $\mathrm{W}_{\text {before }}$ ). Then, the membrane was soaked in abovementioned Fenton's reagent at $80^{\circ} \mathrm{C}$ for $1 \mathrm{~h}$. After that, the membrane was repeatedly washed with DI. water and dried under dynamic vacuum, and then reweight to recode the mass $\left(\mathrm{W}_{\mathrm{after}}\right)$. We evaluated the oxidative stability by the weight loss ratio $\left(\mathrm{W}_{\text {loss }}\right)$ of the membranes after contacting the Fenton reagent by using the equation as follows:

$$
W_{\text {loss }}(\%)=\frac{W_{\text {before }}-W_{\text {after }}}{W_{\text {before }}}
$$




\section{Results and Discussion}

\subsection{Synthesis and Characterization of PVA-ZW polymers}

There are only a handful of examples on covalent functionalization of PVA with zwitterionic groups, and seldom effort has been made to explore these zwitterion modified PVA on the applications of DMFCs membrane. ${ }^{35}$ For instance, zwitterionic groups have been added onto PVA by using tetraethyl orthosilicate as a bridge. The obatained zwitterion modified PVA shows enhanced protin separation. ${ }^{36}$ Huang et al. successfully modified PVA-co-PE nanofiber with zwitterions to achieve improved antifouling function. ${ }^{37}$ Such rarity is due to the inertness of $-\mathrm{OH}$ groups because of the strong inter or intramolecule O-H $\cdots \mathrm{O}$ hydrogen bond. ${ }^{32}$ Known modification strategies for PVA derivation comprise of esterification, etherification and urethanation. The reported esterification reagents generally include acid chlorides, ${ }^{38-39}$ anhydrides,${ }^{40}$ and carboxylic acid active ester. ${ }^{41}$ Concerning the easy hydrolysis of ester bond, PVA etherification via the formation of ether bond has also been developed. The main etherification reagents include epoxide ${ }^{42}$ and alkyl halides based on Williamson synthesis. ${ }^{43}$ Although the ether bond is relatively stable against hydrolysis, harsh conditions (high temperature or highly corrosive reagents) are often necessary for ether bond formation. Urethanation can functionalize PVA under mild conditions. Quantitative urethanation of PVA has been demonstrated by using ionic liquids as solvents and alkyl isocyanate as reagent, ${ }^{38}$ however, the use of ionic liquid is not cost effective. Ossipov, D. A. et al. reported that CDI can effectively activate the hydroxyl groups of PVA at room temperature with controllable derivation degree. ${ }^{32}$ Their strategy allows efficient incorporation of a wide variety of chemical functionalities covalently bonded with PVA. Inspired by their strategy, we first functionalized PVA with amine groups to obtain PVADEEDA- $n$ series, which were then subjected to react with sultone to give zwitterionic groups (see Fig. 1). A milky-white precipitate formed during the reaction as shown in the inset picture of Fig. 3, suggesting that the dense charged groups were added onto the PVA, so that the electrostatic attraction induced the aggregation of PVA chain. The zwitterion-modified PVA, denoted as PVA-ZW-n, is recovered by decanting the DMF 
solvent. The white gel-like products were further purified by repeating dissolution in water and participation in acetone.

Figure 2. The zwitterionic groups substitution degree of PVA as a function of feeding amount of CDI (solid sphere), and $\mathrm{pH}$ of the corresponding aqueous solution. The molar ratios of zwitterionic group to the initial PVA hydroxyl groups ([pendant group]/[-OH $]_{0}$ ) are plotted in Figure 1 as a function of the feed molar ratio of CDI to the hydroxyl group of PVA ([reagent $] /[-\mathrm{OH}]_{0}$ ).

We observed that increasing the feed amount of CDI can efficiently improve the substitution degree of PVA. However, when CDI amount is over 0.5 equiv.-OH of PVA, the reactants solution becomes highly viscous, unsuitable for subsequent treatment. After the stepwise reactions illustrated in Fig. 1, the obtained PVA-ZW polymers can be dissolved in water. In absence of zwitterionic groups, the aqueous solution of PVADEEDA-20 shows a $\mathrm{pH}$ of ca. 10.4. It is interesting to note that the $\mathrm{pH}$ of PVA-ZW solution decreases from the 10.4 of PVA-ZW-0 to ca. 4.4 for PVA-ZW-20. This may imply the acidic strength of sulfonated group is stronger than the conjugated acid of attached $\left(-\mathrm{NEt}_{2}\right)$ amine groups, therefore the aqueous solution of PVA-ZW appears to be acidic; since a certain fraction of sulfonated groups, but not necessarily all, are available to ionize water.

Figure 3. NMR spectra of (a) M-DEEDA, PVA-DEEDA-5 and PVA-DEEDA-20; (b) M-ZW, PVA-ZW-5 and PVA-ZW-20; (c) the proposed configurations of PVA-ZW polymer. Except for M-DEEDA whereby $\mathrm{CDCl}_{3}$ was used as solvent due to its limited solubility in $\mathrm{D}_{2} \mathrm{O}$, the NMR spectra of other samples were recorded in $\mathrm{D}_{2} \mathrm{O}$. The gray circle stands for the segment of polymer with pendent hydroxyl groups. The intensive peak at 4.8 ppm belongs to residual $\mathrm{H}_{2} \mathrm{O}$. Inset picture: observed change in solubility before and after zwitterionic groups bearing. The structural properties of modified polymers were studied by liquid phase ${ }^{1} \mathrm{H}$ NMR with $\mathrm{D}_{2} \mathrm{O}$ as solvent. Considering the relatively complicated chemical environment of the pendent groups and polymeric backbone, the according model compounds were also synthesized to assistant proton assignment. The model compound M-DEEDA was synthesized (Scheme S1) under the same condition as that used in polymer modification. 
Only two sets of $1 \mathrm{H}$ NMR peaks were observed for pristine PVA, which corresponds the $-\mathrm{CH}_{2}$ - and - $\mathrm{CH}$ of main chain, while PVA-DEEDA shows three sets of new characteristic NMR peaks of the tertiary amine $\left(-\mathrm{CH}_{2}-\mathrm{CH}_{2}-\mathrm{NEt}_{2}\right)$. PVA-DEEDA was converted into PVA-ZW by the ring opening of 1,3-propane sultone with the amine of PVA-DEEDA under ambient conditions. The prepared PVA-ZW presents limited solubility in DMF as shown in inset picture of Fig. 3. This can be explained by the formation of polymer aggregates due to the electrostatic or dipole interaction. After converting PVA-DEEDA to PVA-ZW, the methyl groups of $-\mathrm{NCH}_{2}-\mathrm{CH}_{3}$ shifts down field from $1.0 \mathrm{ppm}$ to 1.3 ppm due to the deshielding from the quaternary nitrogen atom.

Interestingly, PVA-ZW-20 with high level substitution degree causes notable distribution of the methylene chain of(- $\left.\mathrm{CH}_{2}(\mathrm{i})-\mathrm{CH}_{2}(\mathrm{j})-\mathrm{CH}_{2}(\mathrm{k})-\mathrm{SO}_{3}{ }^{-}\right)$. In principle, the adjacent segment of $-\mathrm{CH}_{2}(\mathrm{i})-\mathrm{CH}_{2}(\mathrm{j})-\mathrm{CH}_{2}(\mathrm{k})-\mathrm{SO}_{3}{ }^{-}$can be spatially arranged in either parallel or crossed configurations. ${ }^{44}$ Therefore, there are three possible configurations as shown in Fig. 3c, whereby the $-\mathrm{CH}_{2}(\mathrm{i})-\mathrm{CH}_{2}(\mathrm{j})-\mathrm{CH}_{2}(\mathrm{k})-\mathrm{SO}_{3}{ }^{-}$can be either symmetrically arranged (form 1、3) or asymmetrically arranged (form 2). Among these three distribution forms, proton from form 1 should be present at highest frequencies in NMR, while proton in form 2 and form 3 should shift toward low frequencies due to electron shielding from the electron-deficient group of quaternary amine. In comparison to the $\mathrm{CH}_{2}(\mathrm{k})$ - and $-\mathrm{CH}_{2}(\mathrm{j})-$, the $-\mathrm{CH}_{2}(\mathrm{i})$ - atom is closer to the parent PVA backbone, therefore, the spatial position is fixed and the distributed NMR peaks are overlapped, resulting in more complicated splitting patterns ranging from 2.89 to $3.11 \mathrm{ppm}$. Notably, such kind of distribution was not observed for low level substitution degree (5\%), implying that the chain-chain interaction may emerge as the appending extent reaches significant levels. It is worth noting that ionic interaction in PVA-ZW-20 is strong enough to prevent it from dissolution in water. This can be because only very limited PVA-ZW chains hold together in aqueous solution driven by electrostatic force, (For instance, perhaps only PVA-ZW "trimer" or "dimer" formed), and not further aggregation formed, therefore, they can stability "distributed" in water. A similar phenomenon has been observed previously on guanidiniocarbonyl pyrrole carboxylate zwitterion. ${ }^{45}$ 
FTIR was also used to characterize the solid phase PVA-ZW polymers. Certain types of bonds in the zwtterionic pendants, like $\mathrm{S}-\mathrm{O}$ and $\mathrm{C}=\mathrm{O}$, presented as distinctive peaks in FTIR, but could not be effectively reflected in NMR. Representative FTIR spectra are shown in Fig. 4 and the peaks were assigned to the vibration mode according to the DFT calculations (see ESI). Characteristic bands of sulfonate symmetric and asymmetric stretching vibration were clearly observed at 1040 and $1190 \mathrm{~cm}^{-1}$, respectively. The low-intensity peak at $523 \mathrm{~cm}^{-1}$ was due to the bending vibration of S-O bond. S-C starching mode present at $609 \mathrm{~cm}^{-1} \cdot{ }^{46}$ The $-\mathrm{CH}_{2}$ - bonds from the parent PVA could be still clearly observed at $2994-2817 \mathrm{~cm}^{-1}$, but not in the model compound of MZW. These results suggest successful addition of sulfonated groups on parent PVA.

Figure 4. IR spectra (KBr) for neat PVA, M-ZW and PVA-ZW-20. The IR spectra of M-ZW agree well with PVA-ZW-20, implying the appending of zwitterionic groups occurred as expected. The vibration assignments of FTIR are assisted by the DFT calculations (See Fig. S6 in ESI).

\subsection{Membrane Preparation and Characterization}

\subsubsection{PVA-ZW based membrane preparation}

The PVA-ZW polymers can be transformed into transparent membranes (see inset of Figure 1), benefiting from the high molecular weights of the starting PVA polymer.

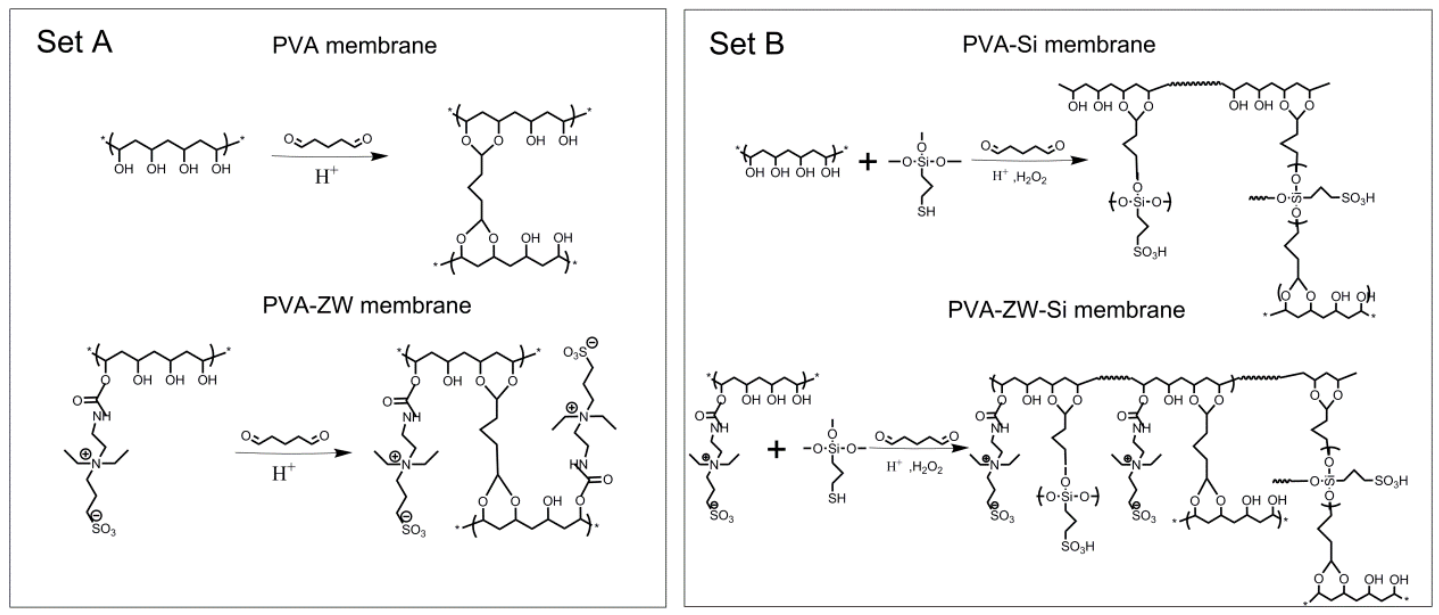


Scheme 1. The experimental sets for the study on zwitterion effects. Set A includes PVA and PVA-ZW membrane; while Set B includes PVA-Si and PVA-ZW-Si membrane.

Table 1. The sample code and the corresponding composites used in the present study.

\begin{tabular}{ccccc}
\hline \multirow{2}{*}{$\begin{array}{c}\text { Composites } \\
(\text { wt. } \%)\end{array}$} & \multicolumn{3}{c}{ PEM sample ID } \\
\cline { 2 - 5 } & PVA & PVA-ZW-20 & PVA-Si & PVA-ZW-20-Si \\
\cline { 2 - 5 } PVA & 100 & 0 & 50 & 0 \\
PVA-ZW-20 & 0 & 100 & 0 & 50 \\
MPDMS & 0 & 0 & 50 & 50 \\
\hline
\end{tabular}

In general, the proton conductivity of solids is positively proportional to the proton density. Considering that proton density in the bulk PVA-ZW membrane is limited, MPDMS, which contains thiol groups that can be further oxidized into sulfonated group, was added into PVA-ZW-20, with the aim of increasing the proton content (see Scheme 1 and experimental section). For comparison, the same doping procedure was also conducted on the neat PVA. We summarized the proton electrolyte membrane (PEM) studied in this work and the corresponding composites code in Table 1. In order to highlight the key role of zwitterionic groups, we conducted the comparison between two sets of experiment (Table 1). Each set includes two types of membranes. The distinct differences between set A and set B are whether zwitterionic groups are incorporated or not.

\subsubsection{Proton Conduction, IEC, Mechanical and Chemical Stability}

Good proton conductivity is necessary for practical DMFCs application. As mentioned previously, two sets of experiments are designed in order to study the influence of zwitterionic groups on proton conduction (Table 1). Proton conduction by a PEM, as observed practically, is a resultant of the in-plane and the through-plane conduction, therefore, we measure both through-plane and in-plane proton conductivity of our membranes. Pristine PVA displayed poor proton conduction as evidenced by the 
extreme high Ohmic resistance (Fig. 5b). Appending zwitterionic groups on PVA promotes ionization of water molecule as indicated by the decrease in $\mathrm{pH}$ with increased pendant zwitterions (see Fig. 2). Therefore, impedance curve of PVA-ZW-20 in Fig. 5a represents the typical proton conduction behavior of PVA-ZW-20 membrane: lower resistance at high frequency attributable to fast proton transportation, with the resistance increasing dramatically at low frequency due to electron blockage at the interface between electrolyte and electrode. According to the Eq. 4, the proton conductivity of PVA-ZW-20 is calculated to be $3 \times 10^{-3} \mathrm{~S} / \mathrm{cm}$, almost ten times higher than that of PVA. However, this is still insufficiently high when compared to that of Nafion 117, which shows $7.5 \mathrm{mS} / \mathrm{cm}$ at $30^{\circ} \mathrm{C}$, and increases to $27.5 \mathrm{mS} / \mathrm{cm}$ at $80^{\circ} \mathrm{C}$ when fully hydrated. In order to enhance the proton conduction, one more set of experiments (Set B, Table 1 and Scheme 1) were conducted, whereby MPDMS was doped in the matrix of PVA and PVA-ZW-20 and subsequently oxidized to give PVA-Si and PVA-ZW-20-Si membrane, respectively (Scheme 1). Both membranes in set B shows enhanced proton conduction when compared to their counterparts in set A. In set B, the proton conductivity of PVA$\mathrm{ZW}-20-\mathrm{Si}$, which contains zwitterionic groups, is $7.3 \mathrm{mS} / \mathrm{cm}$ at $30^{\circ} \mathrm{C}$ and $18 \mathrm{mS} / \mathrm{cm}$ at 80 ${ }^{\circ} \mathrm{C}$, much higher than that of non-zwitterionic PVA-Si. This demonstrates that the zwitterionc groups serve as key role in promoting proton conduction. It is interesting to note that under our experimental condition, the measured proton conduction of Nafion 117 is lower than that reported in literature reported data. ${ }^{47-49}$ This is likely be due to the high contact resistance between electrode and membrane, a consequence of insufficient mechanical pressure of our setup (Fig.S4). It is worth noting that the measurements on all samples were conducted using exactly the same setup, the conclusions drawn are therefore valid.

Figure 5. Typical impedance plot for (a) PVA-ZW-20-Si and (b) PVA at $25{ }^{\circ} \mathrm{C}$ under fully hydrated condition. 
Table 2. The IEC, mechanical stability, and swelling ratio for different membranes.

\begin{tabular}{|c|c|c|c|c|c|}
\hline & & Set A & & Set B & \\
\hline Samples & PVA & PVA-ZW-20 & PVA-Si & PVA-ZW-20-Si & Nafion 117 \\
\hline $\begin{array}{c}\mathrm{IEC}^{\mathrm{a}} \\
\text { (mequiv./g) }\end{array}$ & 0 & 0.56 & 5.0 & 4.3 & 0.91 \\
\hline $\begin{array}{c}\text { Swelling ratio } \\
(\%)\end{array}$ & $20 \%$ & $29 \%$ & $12 \%$ & $15 \%$ & $42 \%$ \\
\hline $\begin{array}{l}\text { Tensile strength } \\
\text { (MPa) }\end{array}$ & 26 & 0.9 & 1.6 & 1 & 21.74 \\
\hline $\begin{array}{c}\text { Elongation at } \\
\text { break }(\%)\end{array}$ & 246.2 & 87.4 & 122 & 54.1 & 90.6 \\
\hline
\end{tabular}

Table 3. The water uptake, proton conduction and oxidative stability for different membranes.

\begin{tabular}{ccccccc}
\hline Samples & & \multicolumn{2}{c}{ Set A } & \multicolumn{3}{c}{ Set B } \\
\cline { 3 - 7 } Properties & PVA & PVA-ZW-20 & PVA-Si & $\begin{array}{c}\text { PVA-ZW- } \\
20-S i\end{array}$ & $\begin{array}{c}\text { Nafion } \\
117\end{array}$ \\
\hline $\begin{array}{c}\text { Conductivity } \\
(\mathrm{mS} / \mathrm{cm})\end{array}$ & $30^{\circ} \mathrm{C}$ & --- & 3 & 2.5 & 7.3 & 7.5 \\
& $80^{\circ} \mathrm{C}$ & --- & 11.1 & 5.5 & 18 & 27.5 \\
$\begin{array}{c}\text { Water sorption } \\
(\text { wt. \%) }\end{array}$ & $30^{\circ} \mathrm{C}$ & $80^{\circ} \mathrm{C}$ & 348 & & 145 & 48 \\
$\begin{array}{c}\text { Oxidative } \\
\text { stability } \\
\text { (wt.\%) }\end{array}$ & $80^{\circ} \mathrm{C}$ & 7.2 & 9.8 & 4.2 & 5.3 & 3.9 \\
\hline
\end{tabular}

Figure 6. Temperature dependent proton conductivities of Nafion 117 and PVA-ZW based membrane at $100 \%$ humidity (full hydration). 
The calculated activation energy values range between 9.6 to $38.5 \mathrm{~kJ} / \mathrm{mol}$, suggesting that all of these membranes conduct proton via Grottuss mechanism. ${ }^{50}$ Among the samples, the conductivity of Nafion 117 is most sensitive to temperature, implying Nafion 117 has the highest activation energy among the studied membranes. This is confirmed by fitting of conduction data $v$ s. temperature using Arrhenius plot. It should be noted that proton conduction in PVA-Si shows much lower activation energy than that in its counterpart PVA-ZW-20-Si, implying that the membrane network is relatively dense due to electrostatic interaction, therefore restricting the proton transportation and increase the $\mathrm{E}_{\mathrm{a}}{ }^{51}$

As shown in Table 2, The zwitterionic polymer PVA-ZW-20 membrane had tensile strength at maximum load in the range of $0.9 \mathrm{MPa}$ and elongation at break of 87.4\%. It was obvious that PVA-ZW-20 has lower elongation ratio than that of PVA, even though the water uptake of PVA-ZW-20 is higher than that of PVA (Table 3). This could be due to the existence of electrostatic interaction, which caused an increased chain packing density and a decrease in the fractional free volume, thereby suppressing linear expansion of the membrane. Upon adding MPTMS, both PVA and PVA-ZW-20 exhibit decreased elongation ratio, with PVA demonstrating much less elongation than PVAZW-20. The reduction in tensile strength and tensile elongation of PVA matrix with the addition of MPTMS might be due to so-called filler effect: the formation of solid particle agglomerates and thus results in a loss in mechanical performance. ${ }^{52}$

The IEC is a widely accepted indicator for the exchangeable ion content in the membrane. The IEC of each dried membrane is studied by exchange of proton with $\mathrm{Na}^{+}$, and then titrated to the endpoint of phenolphthalein with a $\mathrm{NaOH}$ standard solution to quantify the exchanged $\mathrm{H}^{+}$ions. The acid-base interaction which existed in the PVA-ZW based membrane could cause an underestimation of actual IEC, because the proton exchange in the presence of basic groups could be difficult. ${ }^{51}$ This is also supported by the fact that presence of zwitterionic groups results in increased activation energy for proton conduction (Fig. 6). To circumvent this effect, the membrane was kept in the titration solution during the whole titration process, such that the proton dissociation balance can be pushed forward. As expected, the IEC of neat PVA is zero. As a 
consequence of water ionization (Fig. 2), the PVA-ZW-20 membrane shows IEC of 0.53 mequv $\mathrm{g}^{-1}$. Adding MPTMS effectively increased the amount of sulfonated groups, therefore PVA-ZW-20-Si exhibits an IEC of 4.3 mequv $\mathrm{g}^{-1}$. The oxidative stability decrease upon addition of zwitterion in the membrane matrix, maybe because free-radical attack is more aggressive at higher temperatures $\left(80^{\circ} \mathrm{C}\right)$ and occurs in the proximity of hydrophilic domains. ${ }^{53}$

\subsubsection{Methanol permeability, swelling ratio, uptake and selectivity parameter:}

Figure 7. Methanol permeability (9.2M, black) and methanol concentration change after soaking various membranes in $\mathrm{MeOH} /$ water (red) comparison.

$\mathrm{MeOH}$ and water selectivity is evaluated by soaking the membrane in $\mathrm{MeOH} /$ water mixture at room temperature. Nafion117 shows preferential $\mathrm{MeOH}$ adsorption, as indicated by decreased $\mathrm{MeOH}$ concentration as shown in Fig. 7. After PVA-ZW-20 membrane was immersed in $\mathrm{MeOH} /$ water mixture, the $\mathrm{MeOH}$ concentration increases slightly compared to the pristine stock solution, implying that PVA-ZW-20 has a tendency to selectively adsorb water over methanol, perhaps due to alcohol resistance of parent PVA main chain and increased hydrophilicity after adding zwitterionic groups (Table 3). Methanol permeability should generally obey the tendency of methanol/water uptake. Indeed, PVA can effectively block methanol as indicated by its methanol permeability which is the lowest among all tested membranes (Fig. 7, black) The addition of MPTMS slightly increase the methanol blockage performance of neat PVA. Addition of zwitterionic groups results in an increase in an increase in hydrophilicity, thus PVA-ZW-20 and PVA-ZW-20-Si shows increased methanol crossover compared to the pristine PVA, but their ability of methanol blockage still outperforms Nafion 117.

To study the zwitterionic effect in stabilizing dimension, the swelling ratio of each membrane was further investigated after soaking in above-mentioned $\mathrm{MeOH} / \mathrm{H}_{2} \mathrm{O}$ mixture (Table 3). Among these membranes, Nafion shows the highest swelling ratio of 
$\sim 40 \%$, due to its -tendency to adsorb $\mathrm{MeOH} .{ }^{54-55} \mathrm{PVA}$ is a well-known $\mathrm{MeOH}$ barrier, and the crosslinked PVA membrane shows swelling ratio of $23 \%$, indicating its good dimensional stability. As expected, bearing zwitterionic groups on PVA effectively increase its affinity to water, therefore causing the swelling ratio to increase as more water was adsorbed. As Si-O-Si has a very rigid structure, doping with MPTMS can efficiently decrease membrane swelling. As shown in Fig. S7, The enhanced affinity of water can potentially benefit proton conduction at low humidify since a stable water environment and an additional proton conduction pathway can exist within zwitterion modified membrane.

In order to directly compare membrane performance in a systematic way, a key selectivity parameter (SP), namely the ratio of proton conductivity to methanol permeability, is applied to evaluate the tradeoffs.

$$
S P=\frac{\sigma}{P}
$$

Where $\sigma$ is the conductivity, $\mathrm{P}$ is the methanol permeability. Obviously, a higher SP value suggests higher conductivity and lower methanol crossover, placing the membrane among the promising candidates for practical fuel cell applications.

Figure 8. Selectivity parameter of each membrane (calculated according to the $9.2 \mathrm{M} \mathrm{MeOH}$ solution).

Although the PVA-Si, pristine PVA, PVA-ZW membranes are good methanol barrier, their conductivities are relatively poor (Fig. 8). Therefore, their SP values barely outperformed that of Nafion117. PVA-ZW-20-Si shows a SP value up to1.5 times higher than that of Nafion 117, making it a suitable candidate for DMFCs applications. We also have to admit that the mechanical stability of the developed membrane is still modest. Therefore, further effort is in urgent needed to improve the mechanical strength in order to fulfill the practical operation of DMFCs 


\section{Conclusions}

In this study, a series of PVA-based polyzwitterions were synthesized by partially modifying the PVA hydroxyl groups with substitution degree ranging from $5 \%$ to $20 \%$. As suggested by $1 \mathrm{H}$ NMR spectra, when the zwitterionic group content is $20 \%$, the -($\left.\mathrm{NEt}_{2}-\left(\mathrm{CH}_{2}\right)_{3}-\mathrm{SO}_{3}\right)$ groups can be symmetrically or asymmetrically arranged to give three possible configurations. However, no such distribution was observed when the zwitterionic groups substitution degree is 5\%. This phenomenon may imply that the electrostatic interaction is mainly responsible for the observed proton spatial distribution, and low charge density is insufficient to cause electrostatic interaction.

The prepared PVA-ZW polymers can form mechanically robust membranes by themselves, or combined with MPTMS by simple solution casting to form PVA-ZW or PVA-ZW-Si membranes, respectively. The proton conductivity and methanol barrier properties of the synthesized PVA-ZW and PVA-ZW-Si membranes were investigated and compared to those of Nafion and MPTMS doped PVA (PVA-Si). Although PVAZW-20 has no free protons, it exhibits proton conductivity of $3 \mathrm{mS} / \mathrm{cm}$ at $100 \%$ humidity and room temperature. This is perhaps due to its ability to dissociate proton from water, which was suggested by the acidity $(p H<7)$ of PVA-ZW-20 aqueous solution. After addition of $-\mathrm{SO}_{3} \mathrm{H}$ group, PVA-ZW-20-Si shows similar conductivity but much lower methanol permeability when compared to that of Nafion117. The comparison study between PVA-ZW-Si and PVA-Si reveals the positive zwitterion effect, which enhances

proton conductivity. Although mechanical test reveals that appending zwitterion on the main chain of PVA reduces the mechanical performance, PVA-ZW-20-Si still shows sufficient strength and toughness for DMFCs applications.

\section{Acknowledgement:}

This work is financially supported by the start-up funding of "One-hundred Young Talents of Guangdong University of Technology (class A)” program, National Natural Science Foundation of China (Nos. 21372051). The Science and Technology Planning Project of Guangdong Province, China (2016A010103031). 


\section{References}

1.Hardman, S.; Chandan, A.; Steinberger-Wilckens, R.; Fuel cell added value for early market applications. J Power Sources 2015, 287, 297-10.

2.Wannek, C.; Glüsen, A.; Stolten, D.; Materials, manufacturing technology and costs of fuel cell membranes. Desalination 2010, 250, 1038-4.

3.Aricò, A. S.; Srinivasan, S.; Antonucci, V., DMFCs: From Fundamental Aspects to Technology Development. Fuel Cells 2001, 1 (2), 133-29.

4. Li, Q.; Wang, T.; Havas, D.; Zhang, H.; Xu, P.; Han, J.; Cho, J.; Wu, G.; HighPerformance Direct Methanol Fuel Cells with Precious-Metal-Free Cathode. Adv. Sci.2016, 1600140-10.

5.Ahmed, M.; Dincer, I.; A review on methanol crossover in direct methanol fuel cells: challenges and achievements. Int. J. Energy Res.2011, 35, 1213-16.

6.P. Kumar, K.; Dutta, S.; Das, P.; Paban K.; An overview of unsolved deficiencies of direct methanol fuel cell technology: factors and parameters affecting its widespread use , Int. J. Energy. Res. 2014, 38, 1367-24.

7. Kamarudina, S.K.; Achmada, F.; Dauda, W.R.W. ; Overview on the application of direct methanol fuel cell (DMFC) for portable electronic devices, Int. J. Hydro. Energy, 2009, 34, 6902-15.

8.Kreuer, K. D., On the development of proton conducting polymer membranes for hydrogen and methanol fuel cells. J Membrane Sci 2001, 185 (1), 29-11.

9.H. Zhang and P. K. Shen, Recent development of polymer electrolyte membranes for fuel cells Chem. Rev., 2012, 112 (5), 2780-53.

10. Wu, L.; Zhang, Z.; Ran, J.; Zhou, D.; Li, C.; Xu, T., Advances in proton-exchange membranes for fuel cells: an overview on proton conductive channels. Phys. Chem. Chem. Phys. 2013, 15 (14), 4870-18.

11. H. Zhang, H. Huang, P.K. Shen, Methanol-blocking nafion composite membranes fabricated by layer-by-layer self-assembly for direct methanol fuel cells, Int. J. Hydrog. Energy 2012, 37, 6875-5.

12. Liu, J.; Wang, H.; Cheng, S.; Chan, K.-Y., Nafion-polyfurfuryl alcohol nanocomposite membranes with low methanol permeation. Chem. Commun. 2004, 6, 728-2.

13. Mahajan, C. V.; Ganesan, V., Influence of Hydrogen Bonding Effects on Methanol and Water Diffusivities in Acid-Base Polymer Blend Membranes of Sulfonated 
Poly(ether ether ketone) and Base Tethered Polysulfone. J. Phys. Chem. B 2013, 117 (17), 5315-15;

14. Wu, L.; Huang, C.; Woo, J.-J.; Wu, D.; Yun, S.-H.; Seo, S.-J.; Xu, T.; Moon, S.-H., Hydrogen Bonding: A Channel for Protons to Transfer through Acid-Base Pairs. J. Phys. Chem. B 2009, 113 (36), 12265-6;

15. Wu, L.; Huang, C.; Woo, J.-J.; Wu, D.; Yun, S.-H.; Seo, S.-J.; Xu, T.; Moon, S.-H., Modifying a Proton Conductive Membrane by Embedding a "Barrier". J. Phys. Chem. B 2010, 114 (41), 13121-7;

16. Wu, L.; Zhou, D.; Wang, H.; Pan, Q.; Ran, J.; Xu, T., Ionically Cross-Linked Proton Conducting Membranes for Fuel Cells. Fuel Cells 2015, 15 (1), 189-7;

17. Fu, R.-Q.; Julius, D.; Hong, L.; Lee, J.-Y., PPO-based acid-base polymer blend membranes for direct methanol fuel cells. J Membrane Sci 2008, 322 (2), 331-8.

18. Deimede, V.; Voyiatzis, G. A.; Kallitsis, J. K.; Qingfeng, L.; Bjerrum, N. J., Miscibility Behavior of Polybenzimidazole/Sulfonated Polysulfone Blends for Use in Fuel Cell Applications. Macromolecules 2000, 33 (20), 7609-9.

19. Lowe, A. B.; McCormick, C. L., Synthesis and Solution Properties of Zwitterionic Polymers. Chem. Rev. 2002, 102, 4177-14.

20. Zhang, Q.; Zhang, S.; Li, S., Synthesis and characterization of novel cardo poly(aryl ether sulfone) bearing zwitterionic side groups for proton exchange membranes. Int $\mathbf{J}$ Hydrogen Energ 2011, 36 (9), 5512-9.

21. Jamiu, Z. A.; Saleh, T. A.; Ali, S. A., Synthesis of a unique cross-linked polyzwitterion/anion with an aspartic acid residue and its use for $\mathrm{Pb}^{2+}$ removal from aqueous solution. RSC Adv. 2015, 5, 42222-11.

22. Schlenoff, J. B., Zwitteration: Coating Surfaces with Zwitterionic Functionality to Reduce Nonspecific Adsorption. Langmuir 2014, 30, 9625-12;

23. Shao, Q.; Jiang, S., Molecular Understanding and Design of Zwitterionic Materials. Adv.Mater. 2015, 27 (1), 15-12.

24. Kobayashi, M.; Takahara, A., Environmentally friendly repeatable adhesion using a sulfobetaine-type polyzwitterion brush. Polym. Chem. 2013, 4 (18), 4987-6.

25. Tiyapiboonchaiya, C.; Pringle, J. M.; Sun, J.; Byrne, N.; Howlett, P. C.; MacFarlane, D. R.; Forsyth, M., The zwitterion effect in high-conductivity polyelectrolyte materials. Nat Mater 2004, 3 (1), 29-4. 
26. Gan, L. M.; Chow, P. Y.; Liu, Z.; Han, M.; Quek, C. H., The zwitterion effect in proton exchange membranes as synthesised by polymerisation of bicontinuous microemulsions. Chem. Commun. 2005, 35, 4459-3.

27. Brown, R. H. et al. Effect of ionic liquid on mechanical properties and morphology of zwitterionic copolymer menbranes. Macromolecules 2010, 43, 790-7.

28. Peng, X. et al. A zwitterionic gel electrolyte for efficient solid-state supercapacitors. Nat. Commun.2016, 7:11782 doi: 10.1038/ncomms11782.

29. Li, Y.; Lin, X.; Wu, L.; Jiang, C.; Hossain, M. M.; Xu, T., Quaternized membranes bearing zwitterionic groups for vanadium redox flow battery through a green route. $\mathrm{J}$ Membrane Sci 2015, 483, 60-10;

30. Xu, J.; Cheng, H.; Ma, L.; Han, H.; Wang, Z., Construction of a new continuous proton transport channel through a covalent crosslinking reaction between carboxyl and amino groups. Int J Hydro. Energy 2013, 38 , 10092-12;

31. Chu, F.; Lin, B.; Feng, T.; Wang, C.; Zhang, S.; Yuan, N.; Liu, Z.; Ding, J., Zwitterion-coated graphene-oxide-doped composite membranes for proton exchange membrane applications. J Membrane Sci. 2015, 496, 31-8.

32. Ossipov, D. A.; Hilborn, J., Poly(vinyl alcohol)-Based Hydrogels Formed by "Click Chemistry". Macromolecules 2006, 39, 1709-10.

33. Will, B.; Lichtenthaler, R. N., Comparison of the separation of mixtures by vapor permeation and by pervaporation using PVA composite membranes. I. Binary alcoholwater systems. J Membrane Sci. 1992, 68, 119-7.

34.Margolese, D.; Melero, J. A.; Christiansen, S. C.; Chmelka, B. F.; Stucky, G. D., Direct Syntheses of Ordered SBA-15 Mesoporous Silica Containing Sulfonic Acid Groups. Chem. Mater. 2000, 12 (8), 2448-12.

35. Tripathi, B. P.; Shahi, V. K., 3-[[3-(Triethoxysilyl)propyl]amino]propane-1-sulfonic Acid-Poly(vinyl alcohol) Cross-Linked Zwitterionic Polymer Electrolyte Membranes for Direct Methanol Fuel Cell Applications. ACS Appl. Mater. Interfaces, 2009, 1, 1002-13.

36. Liu, G. , Zhang, L., Mao, S.; Rohanic, S.; Ching, C.; Lu. J.; Zwitterionic chitosansilica-PVA hybrid ultrafiltration membranes for protein separation. Sep. Purif. Technol. 2015, 152, 55-9.

37. Huang, J.; Wang, D.; Lu, Y.; Li, M.; Xua, W.; Surface zwitterionically functionalized PVA-co-PE nanofiber materials by click chemistry. RSC Adv., 2013,3, 20922-8. 
38.Eastman, S. A.; Lesser, A. J.; McCarthy, T. J., Quantitative Poly(vinyl alcohol) Modification in Ionic Liquids: Esterification and Urethanation with Low Surface Tension Producing Reagents. Macromolecules 2010, 43, 4584-5.

39. Orienti, I.; Bigucci, F.; Gentilomi, G.; Zecchi, V., Self-assembling poly(vinyl alcohol) derivatives, interactions with drugs and control of release. J. Pharm. Sci. 2001, 90, 143510.

40. Martens, P.; Holland, T.; Anseth, K. S., Synthesis and characterization of degradable hydrogels formed from acrylate modified poly(vinyl alcohol) macromers. Polymer 2002, 43, 6093-8.

41.Sharma, S. D.; Granberry, M. E.; Jiang, J.; Leong, S. P. L.; Hadley, M. E.; Hruby, V. J., Multivalent Melanotropic Peptide and Fluorescent Macromolecular Conjugates: New Reagents for Characterization of Melanotropin Receptors. Bioconjugate Chem 1994, 5, $591-11$.

42.Martens, P.; Anseth, K. S., Characterization of hydrogels formed from acrylate modified poly(vinyl alcohol) macromers. Polymer 2000, 41, 7715-8.

43.Breitenbach, A.; Jung, T.; Kamm, W.; Kissel, T., Biodegradable comb polyesters containing polyelectrolyte backbones facilitate the preparation of nanoparticles with defined surface structure and bioadhesive properties. Polym. Adv. Tec. 2002, 13, 938-13.

44.Gao, Y.; Robertson, G. P.; Guiver, M. D.; Mikhailenko, S. D.; Li, X.; Kaliaguine, S., Synthesis of Copoly(aryl ether ether nitrile)s Containing Sulfonic Acid Groups for PEM Application. Macromolecules 2005, 38 (8), 3237-9.

45. Schmuck, C.; and Wienand, W.; Highly Stable Self-Assembly in Water: Ion Pair Driven Dimerization of a Guanidiniocarbonyl Pyrrole Carboxylate Zwitterion. J. Am. Chem. Soc., 2003, 125 (2), 452-8.

46.Qian, W.; Krimm, S., Conformation dependence of the SH and CS stretch frequencies of the cysteine residue. Biopolymers 1992, 32 (11), 1503-16.

47. Ma, L.; Li, J.; Cai, W.; Fan, K.; Jiang, Y.; Cheng, H., A facile method to construct highly efficient methanol resistive polyamide-based proton exchange membrane, Int J Hydro. Energy 2016, doi:10.1016/j.ijhydene.2016.04.218. 
48. Lee, C. H.; Park, H. B.; Lee, Y. M.; Lee, R. D., Importance of Proton Conductivity Measurement in Polymer Electrolyte Membrane for Fuel Cell Application. Ind. Eng.

Chem. Res. 2005, 44 (20), 7617-10.

49.Maréchal, M.; Souquet, J. L.; Guindet, J.; Sanchez, J. Y., Solvation of sulphonic acid groups in Nafion ${ }^{\circledR}$ membranes from accurate conductivity measurements. Electrochem Commun 2007, 9 (5), 1023-6.

50.Hara, N.; Ohashi, H.; Ito, T.; Yamaguchi, T., Rapid Proton Conduction through Unfreezable and Bound Water in a Wholly Aromatic Pore-Filling Electrolyte Membrane. J. Phys. Chem. B 2009, 113, 4656-8.

51.Ye, Y.-S.; Chen, W.-Y.; Huang, Y.-J.; Cheng, M.-Y.; Yen, Y.-C.; Cheng, C.-C.; Chang, F.-C., Preparation and characterization of high-durability zwitterionic crosslinked proton exchange membranes. J Membr. Sci 2010, 362 (1-2), 29-9.

52. Tripathi, B. P. and Shahi, V. K.; 3-[[3-(Triethoxysilyl)propyl]amino]propane-1sulfonic Acid-Poly(vinyl alcohol) Cross-Linked Zwitterionic Polymer Electrolyte Membranes for Direct Methanol Fuel Cell Applications, ACS Appl. Mater. Inter., 2009, 1, $5,1002-11$.

53. Asano, N.; Akoi, M.; Suzuki, S.; Miyatake, K.; Uchida, H.; Watanabe, M. J. Am. Chem. Soc. 2006, 128, 1762-8.

54.Hung-Chung Chien, Li-Duan Tsai, Chiu-Ping Huang, Chi-yun Kang, Jiunn-Nan Lin, Feng-Chih Chang Sulfonated graphene oxide/Nafion composite membranes for highperformance direct methanol fuel cells. Int. J. Hydro. Energy 2013, 38, 13792-10.

55. Zhao, C.; Lin, H.; Zhang, Q.; Na, H.; Layer-by-layer self-assembly of polyaniline on sulfonated poly(arylene ether ketone) membrane with high proton conductivity and low methanol crossover, Int. J. Hydro. Energy 2010, 35, 10482-7. 